\title{
GEOTECNOLOGIAS APLICADAS À GEOLOGIA
}

\section{CARLOS ROBERTO DE SOUZA FILHO \& ALVARO PENTEADO CRÓSTA}

Geotecnologia reúne o conjunto de ciências e tecnologias relacionadas à aquisição, armazenamento em bancos de dados, processamento e desenvolvimento de aplicações utilizando informações geo-referenciadas (ou geo-informações). De modo mais específico, ela engloba, de forma isolada ou em conjunto, o Sensoriamento Remoto, a Cartografia Digital, os Sistemas de Informações Geo-Referenciadas, a Aerogeofísica e a Geoestatística.

O Sensoriamento Remoto agrega tecnologias de sensores imageadores e também não imageadores. Sensores portáteis não imageadores, ou espectrorradiômetros, realizam densa amostragem do espectro eletromagnético (com milhares de bandas espectrais de largura nanométrica), abrangendo os comprimentos de onda do visível (VIS), do infravermelho próximo (NIR), do infravermelho de ondas curtas (SWIR) e do infravermelho termal (TIR). Esses sensores são críticos para o sucesso das aplicações que envolvem a caracterização espectral in situ de materiais geológicos e para a simulação da detecção desses alvos por sensores imageadores multiespectrais de baixa resolução espectral (< dezenas de bandas), de alta resolução espectral ( $>10$ bandas) e sensores imageadores hiperespectrais (dezenas a centenas de bandas).

A evolução tecnológica dos sensores imageadores aponta para a disponibilidade futura de sensores ultraespectrais, os quais terão capacidade de adquirir dados em milhares de bandas espectrais e com sofisticação suficiente para reproduzir o comportamento dos alvos, como atualmente medidos pelos espectrorradiômetros.

Quanto aos sensores multiespectrais orbitais com cobertura global, há atualmente várias possibilidades de escolha entre aqueles que operam no espectro de energia refletida e emitida, levando em conta a necessidade de cada tipo de aplicação e a tecnologia disponível. O marco inicial do sensoriamento remoto orbital se deu em 1972, com o lançamento do primeiro satélite de recursos terrestres da série Landsat em órbita terrestre, tendo a bordo o sensor Multiespectral Scanner (MSS), com 4 bandas espectrais cobrindo o VIS e o NIR. Em 1982, o sensor Thematic Mapper, a bordo do Landsat-4, revolucionou o sensoriamento remoto, com a possibilidade de geração de informações em 7 bandas, no VIS, NIR, SWIR e TIR. Uma análise do programa Landsat feita pela NASA, por ocasião da celebração dos seus 30 anos, revelou o grande impacto desse programa nas Ciências da Terra, sendo a Geologia uma das grandes beneficiárias. Em 1990, o sensor OPS a bordo do satélite JERS-1 avançou na capacidade de detecção no espectro SWIR, expandindo a faixa de cobertura da banda 7 do TM e subdividindo-a em 3 bandas independentes. Em 1999 foi lançado o sensor ASTER, a bordo da plataforma TERRA. O ASTER consiste de três sub-sistemas de imageamento independentes, os quais coletam dados em 14 bandas individuais no espectro eletromagnético: (i) a região do VIS-NIR com 3 bandas espectrais, na resolução espacial de 15m; (ii) a região do SWIR, com 6 bandas, na resolução de $30 \mathrm{~m}$; e (iii) e a região do TIR com 5 bandas, na resolução de $90 \mathrm{~m}$ (único sensor orbital capaz de proporcionar imageamento multiespectral termal - diurno e noturno). Em 2000, entrou em operação o Hyperion, primeiro sensor orbital hiperespectral a bordo da plataforma Earth Observing (EO-1), com 220 bandas entre o VIS-SWIR $(0.4 \mathrm{a} 2.5 \mu \mathrm{m})$, na resolução espacial de $30 \mathrm{~m}$.

$\mathrm{Na}$ faixa das microondas, onde operam os sensores ativos de radar, a evolução foi igualmente acentuada. Iniciando com o Seasat em 1978, uma série de radares orbitais entrou em operação nas décadas posteriores. Este primeiro sistema orbital de radar foi seguido pelos experimentos com os sistemas SIR-A (1981) e SIR-B (1984), a partir do ônibus espacial da NASA, que evidenciaram o grande potencial de informações geológicas contidas nas imagens de radares orbitais de abertura sintética (SAR). Sensores SAR passaram a operar rotineiramente a partir de 1991, com o lançamento do ERS-1 pela agência espacial européia, contando com um radar operando na banda $\mathrm{C}$, com polarização VV e resolução espacial de 30m. Em 1992 foi lançado o JERS-1 que, além do sensor OPS, contava também com um sensor SAR operando na banda L, com polarização HH e resolução espacial de $18 \mathrm{~m}$. Estas características do JERS-1/SAR, combinadas com um ângulo de visada de $35^{\circ}$, logo tornaram-no importante fonte de dados para interpretação geológica, notadamente em áreas tropicais como a Amazônia. Em 1995, foi lançado o Radarsat-1, que incorporou avanços tecnológicos significativos, tais como a possibilidade de múltiplas configurações de resolução e ângulos de visada. Em 2002 entrou em operação o Envisat, contando com múltipla polarização (VV e HH). A tendência de evolução tecnológica observada nos radares orbitais aponta para a disponibilidade de sistemas multipolorização e multi-frequiência, de forma similar à evolução já experimentada pelos sensores óticos.

Assim, num período de cerca de 30 anos, a partir da cobertura de um intervalo restrito do espectro (VIS-NIR) com 4 bandas espectrais, os sensores orbitais evoluíram, passando a abranger o SWIR, TIR e as microondas, além de passarem a contar com até centenas de bandas espectrais no caso dos sensores óticos, significando um ganho de resolução espectral da ordem de 50 vezes. Um aumento dessa magnitude na resolução e cobertura espectral tem reflexos diretos na caracterização da assinatura espectral de alvos de interesse geológico, bem como na extração de atributos geológicos dos terrenos. Nas microondas, a introdução de sensores orbitais com propriedades de multi-polorização e multi-freqüência deverá trazer benefícios consideráveis para as aplicações geológicas.

Obviamente, a identificação de feições geológicas na superfície não depende exclusivamente da resolução espectral, mas também da resolução espacial desses sensores. No sensor MSS, a resolução espacial era limitada a 80m. Em 1982, o sensor TM introduziu uma resolução de 30m, superada em 1986 pelo sensor SPOT, com 10m. Em 1999 iniciou-se uma nova era de sensores comerciais de alta resolução espacial, com o lançamento do sensor IKONOS, com até $1 \mathrm{~m}$ de resolução espacial. Em 2001, a barreira de $1 \mathrm{~m}$ de resolução foi quebrada pelo sensor QuickBird, com resolução 
máxima de $0,61 \mathrm{~m}$. Assim, em aproximadamente 30 anos, observouse um ganho em resolução espacial da ordem de 130 vezes.

Em relação aos sensores imageadores aeroportados, além da resolução espacial, variável em função da altura do vôo, a tendência recente é de uso progressivo de sensores hiperespectrais, baseados no formato e nas especificações do sensor AVIRIS da NASA (com 224 bandas entre o VIS-SWIR) - embora dados adquiridos por sensores multiespectrais de alta resolução, como o GEOSCAN (24 bandas entre o VIS-TIR), proporcionem bons resultados em aplicações geológicas. Na categoria de sensores hiperespectrais em uso, há o HYDICE (210 bandas - VIS-SWIR), o PROBE-1 (128 bandas - VIS-SWIR), o CASI (até 228 bandas VIS-NIR), o HyMap (100-200 bandas - VIS-TIR), o AISA (até 288 bandas-VIS-NIR), e os sensores DAIS 7915, DAIS 21115, EPS-H (com 79 a 211 bandas entre o VIS-TIR). Assim como nos sensores orbitais, os radares imageadores aeroportados contam atualmente com multi-frequência, multi-polarização e multi-resolução. Por exemplo, o SAR a bordo da aeronave EMB -145/R99-B do Sistema de Proteção da Amazônia (SIPAM) possui multi-visada, opera nas bandas Le X e tem vários modos de resolução espacial (18, 6 e 3 m) e contempla ainda polarizações HH, HV, VH e VV. Nessa aeronave, existe também um sensor multiespectral, denominado Multispectral Scanner (MSS), que opera com 11 ou 31 bandas distribuídas entre o VIS e o TIR $(0.42-12.5 \mu \mathrm{m})$ e com campos de visada instantânea (IFOV) de 1.25 e 2.5 graus. O SIPAM conta ainda com um sensor multiespectral de alta resolução denominado HSS, com 50 bandas entre o VIS e o TIR $(0.445-12.08 \mu \mathrm{m})$.

Ainda no tocante a sensores passivos aeroportados, há uma convergência de interesses em explorar os não-imageadores de perfilamento hiperespectral que operam no VIS-SWIR e no TIR. O TIPS (Thermal Infrared Line Profiling Spectrometer) é um sensor baseado no instrumento FTIR (Fourier Thermal Infrared), que registra radiação em cerca de 100 canais compreendidos na faixa entre 8-13microns, com pixels de $10 \mathrm{~m}$ (para uma altitude do avião de $100 \mathrm{~m}$ ). A maior parte dos silicatos, incluindo quartzo e feldspatos, e dos carbonatos, possuem assinaturas espectrais características nesse intervalo de comprimentos de onda, motivo do interesse cada vez maior da exploração de sensores termais imageadores e não-imageadores. O OARS (Operational Airborne Research Spectrometer) é outro sensor de perfilagem hiperespectral que adquire dados em 190 canais na faixa entre o VIS e o SWIR, com resolução espacial de $8 \mathrm{~m}$ para uma altitude nominal de vôo de $80 \mathrm{~m}$. O OARS tem sido utilizado ainda em plataformas multi-propósito, onde é integrado a sensores geofísicos para a aquisição simultânea de dados magnéticos, gamaespectrométricos e de reflectância espectral, co-registrados.

Avanços expressivos vêm sendo feitos também em Cartografia Digital, principalmente com o uso de estereoscopia orbital. Vários programas se destacam nesse segmento: SPOT, ASTER, Ikonos e QuickBird, todos com estereoscopia óptica convencional; o RADARSAT-1 com estereoscopia por visada oposta e/ou por visadas com diferentes ângulos e a missão do Shuttle Radar Topographic Mission (SRTM), com estereoscopia por radar interferométrico.

O sistema VIS-NIR do ASTER conta com dois telescópios, um dos quais opera com retro-visada ao longo da órbita do satélite, com poucos segundos de diferença da visada nadir, o que permite gerar pares estereoscópicos. A partir desses pares é possível gerar modelos digitais de elevação (MDEs) com precisão absoluta de até $7 \mathrm{~m}$ (vertical e horizontal, com uso de pontos de controle no terreno), precisão relativa de até $10 \mathrm{~m}$ (vertical e horizontal, sem pontos de controle), e bases topográficas compatíveis com escala 1:50.000 ou maior. O RADARSAT-1, com suas variações em azimute de visada e incidência, tem sido utilizado como provedor de MDEs de alta resolução espacial (modo Fine - resolução $10 \mathrm{~m}$ ), permitindo a geração de cartas topográficas que permitem mapeamento de pelo menos 1:100.000. Os dados da Shuttle Radar Topography Mission foram o resultado de uma missão espacial internacional realizada em 2000, que envolveu a NASA (National Aeronautics and Space Adminis-tration), a NIMA (National Imagery and Mapping Agency), o USDD (United States Department of Defense), a DLR (Centro Aeroespacial Alemão) e a ASI (Agência Espacial Italiana), com o objetivo de gerar um MDE de alta resolução da Terra empregando da interferometria de radar. Toda a base da SRTM consiste de imagens com resolução espacial entre 1 arco-segundo (= 30 m no Equador) e 3 arco-segundos (= $90 \mathrm{~m}$ no Equador), em latitude e longitude. A base de maior resolução permite a geração de MDEs com precisão compatível com mapas topográficos na escala de pelo menos 1:100.000 ou superior.

Os modelos de análise espacial desenvolvidos em Sistemas de Informações Geo-Referenciadas (SIGs) visam combinar dados espaciais para descrever e analisar interações, de modo a fazer previsões por meio de modelos prospectivos empíricos, que forneçam apoio para a definição de sítios de maior potencial para a ocorrência de depósitos minerais, ou de outros fenômenos geológicos. A combinação de dados multi-fontes permite reduzir a ambigüidade de interpretação que normalmente ocorre na análise individual dos mesmos. Os modelos de análise espacial podem ser divididos em data-driven e knowledge-driven. No modelo datadriven os vários mapas de entrada são combinados por diferentes técnicas, tais como, regressão logística, ponderação por evidências (teorema bayesiano), razões de probabilidade e redes neurais (inteligência artificial). Os modelos knowledge-driven incluem o uso da lógica booleana (ou lógica crisp), média ponderada, lógica difusa (nebulosa) ou possibilidade fuzzy, e teoria da crença de Dempster-Shafer. Cada vez mais, os avanços em SIG situam-se não somente na disponibilidade de novas técnicas de análise espacial de dados e de algoritmos matemáticos mas, principalmente, na conversão destes em programas com interação mais simples e amigável com o usuário final. Dois software vêm se destacando por cumprirem requisitos simultâneos de robustez e facilidade de uso: o Arc-Spatial Data Modeler e o GEODAS. O Arc-SDM foi desenvolvido por G. Bonham-Carter/L. Kemp (GSC), em conjunto com G. Raines (USGS), sob patrocínio dos serviços geológicos do Canadá e EUA e empresas de mineração. A primeira versão do software é disponibilizada gratuitamente em http://ntserv.gis. nrcan.gc.ca/sdm/. O ArcSDM fornece as ferramentas para aplicação de vários métodos de análise espacial na avaliação de favorabilidade mineral, entre os quais a ponderação por evidências (WofE - weights of evidence), regressão logística, possibilidade fuzzy e redes neurais. O GEODAS (Geo Data Analysis System), desenvolvido por Q. Cheng (York University, Canada) em colaboração com G. Bonham-Carter (GSC) e G. Raines (USGS), se distingue por ferramentas únicas de processamento e análise estatística de dados, como a interpolação por krigagem e por fractais, regressão múltipla e análises de agrupamento, discriminante e por redes neurais, wavelet e U-statistics. Entretanto, é um software de domínio fechado (http://www. gisworld.org/geodas/), disponível apenas às instituições e empresas que custeiam seu desenvolvimento.

A Aerogeofísica é um dos ramos das Geotecnologias que experimentou um dos maiores desenvolvimentos nos últimos anos, com contribuições importantes para o avanço do conhecimento 
geológico e exploração mineral em todos tipos de terreno. Entre os seus desenvolvimentos recentes destacam-se: (i) sistematização dos levantamentos magnéticos, gamaespectrométricos, eletromagnéticos (no domínio da frequiência e do tempo), com alta resolução e densidade de amostragem (espaçamento de linhas de vôo de $250 \mathrm{~m}$ ou inferior); (ii) sofisticação e incremento dos instrumentos para controle da posição e altura do vôo e dos equipamentos geofísicos, incluindo altimetria a laser; (iii) utilização de sistemas de alta resolução, sensibilidade e freqüência de amostragem; (iv) melhorias nos padrões e procedimentos de calibração; (v) incentivo para o desenvolvimento de técnicas de aquisição e processamento de dados aerogravimétricos; (vi) avanços no préprocessamento (e.g., micronivelamento, que trouxe grandes benefícios com a remoção dos ruídos causados pelo desnivelamento das linhas de vôo) e processamento (e.g., deconvolução de Euler para delineamento de estruturas em profundidade; terraceamento; índice de favorabilidade para urânio; potássio anômalo) de dados; (vii) software robustos e mais amigáveis para processamento de dados.

Essa evolução da aerogeofísica vem trazendo grandes benefícios para regiões de clima tropical, como o Brasil, onde os processos supergênicos levam à formação de solos espessos e a cobertura vegetal impede o acesso a informações sobre o substrato. Ademais, em um país com dimensões continentais e forte carência de mapeamentos geológicos básicos, a análise integrada de dados aerogeofísicos e de sensoriamento remoto tem muito a contribuir no avanço do conhecimento geológico do território e na delimitação de áreas potenciais para a ocorrência de depósitos minerais.

A Geoestatística vem se destacando como uma das técnicas modernas de análise e modelagem espacial, cujo emprego tem trazido impactos positivos para diversas áreas das Geociências. Uma das vantagens reside na sua capacidade de possibilitar a elaboração de modelos espaciais, mesmo em situações onde a quantidade de informações espaciais é restrita e o objeto de estudo é caracterizado por heterogeneidades, como o é a maioria dos corpos mineralizados, reservatórios de hidrocarbonetos e mesmo formações geológicas.

Exposto este breve panorama do estado da arte das Geotecnologias em nível mundial, faz-se necessário considerar como as mesmas vêm sendo empregadas e disseminadas no Brasil, bem como de seus benefícios e impactos para o desenvolvimento das ciências geológicas em nosso país. O objetivo deste número temático é justamente o de apresentar um panorama para o Brasil. Para tanto, foram enviados convites pelos editores à comunidade atuante na área das Geotecnologias, com excelente resposta. Foram recebidos 30 artigos no total, 24 dos quais foram selecionados após criterioso processo de revisão por pares e compõem o presente número temático da Revista Brasileira de Geociências. Estes artigos trazem uma amostra representativa e abrangente dos resultados significativos que vêm sendo obtidos por pesquisadores brasileiros nesse campo das ciências.

Os artigos de Almeida $\boldsymbol{e t}$ al. e Swalf $\boldsymbol{e}$ t $\boldsymbol{a l}$. apresentam uma interessante combinação entre caracterização espectral de alvos por meio de espectrorradiometria e predição de como esses alvos podem ser diferenciados em imagens de Sensoriamento Remoto. Eles desenvolveram modelos de prospecção com base na assinatura espectral dos alvos de interesse, respectivamente, para depósitos de $\mathrm{Zn}-\mathrm{Pb}$ do tipo estratiforme hospedados em rochas metassedimentares na região de Porteirinha (MG), e para depósitos de Au encaixados em xistos carbonosos na região de Paracatu (MG). Esses modelos foram aplicados a imagens do sensor aeroportado GEOSCAN (24 bandas, entre 450nm e 12.000nm ) para os depósitos de $\mathrm{Zn}-\mathrm{Pb}$, e a imagens do sensor TM (6 bandas no espectro refletido) para o depósitos de Au. No primeiro caso, foi possível identificar o corpo principal do minério, em função da resolução espacial de $5 \mathrm{~m}$ e espectral de 24 bandas do sensor. França \& Souza Filho, num estudo multi-temporal de imagens TM e ETM+ adquiridas entre 1986 e 2001 sobre a costa leste da Ilha de Marajós, mapearam áreas progradacionais e retrogradacionais, identificando a direção e distribuição espacial das mudanças e setores costeiros sujeitos à erosão e acresção. Almeida et al., utilizando imagens do sensor TM do Landsat-5 e uma estratégia de processamento orientada para extração de informações específicas da paisagem das lagoas do Pantanal da baixa Nhecolândia (MS), conseguiram correlacionar o parâmetro alcalinidade/salinidade dos corpos d'água com a disposição das cordilheiras e vazantes, fornecendo importantes subsídios para a interpretação da gênese deste ambiente.

Quanto às contribuições em Cartografia Digital, Paradella $\boldsymbol{e t}$ al. apresentam resultados sobre a produção de cartas topográficas por estereoscopia de alta resolução do RADARSAT e sua integração com dados do sensor TM, demonstrando que a acurácia planialtimétrica dos produtos cartográficos obtidos atendem aos requisitos de mapeamentos 1:100.000 e que a tecnologia de RADAR orbital pode ser utilizada com sucesso para mapeamento de semi-detalhe na Amazônia. A estereoscopia SAR também é o tema do trabalho de Santos et al., que discutem aspectos relacionados às estereoscopias de radar (RADARSAT) e híbrida (RADARSAT \& TM/Landsat-5) e suas performances para o mapeamento geológico na região Amazônica (Carajás). Chaves et al. abordam o sinergismo entre dados gerados por vários sensores de radar (RADARSAT, JERS-1, GEMS-1/Randambrasil) e imagens do TM/Landsat-5, com resultados positivos para o mapeamento geológico da região de Bezerra-Cabeceiras (GO).

O uso de Sistemas de Informações Geo-referenciadas na prospecção de recursos minerais é o tema de cinco artigos neste número temático. Moreira et $\boldsymbol{a l}$. comparam o desempenho de vários métodos de análise multi-critério de dados geológicos e de radiometria gama, na predição de áreas potenciais à ocorrência de minerais radiativos no planalto de Poços de Caldas. Os métodos que apresentaram os melhores resultados em foram a ponderação por evidências, possibilidade fuzzy-pah (processo analítico hierárquico) e o modelo com média ponderada. Rocha et al. abordam métodos similares, aplicados à prospecção de $\mathrm{Pb}-\mathrm{Zn}$ na bacia de Irecê (BA). Os autores utilizam geraram mapas evidenciais baseados em um modelo exploratório com base em dados geoquímicos, logrando prognosticar os depósitos com base na análise espacial knowledge-driven (possibilidade fuzzy) e data-driven (WofE). Também na Bahia, na Folha de Itaberaba, Nóbrega \& Souza Filho utilizaram um banco de dados multi-propósito e dados de ocorrências de ocorrências minerais para gerar mapas de favorabilidade com o auxílio das redes neurais, fazendo uso da tecnologia de inteligência artificial. Os resultados foram interessantes, mas de difícil interpretação face à ausência de uma quantidade maior de depósitos e ocorrências conhecidas. Silva et al. apresentam uma técnica inovadora para análise espacial de dados, denominada razões de probabilidade. A técnica, aplicada a dados aerogeofísicos, apontou novas áreas com potencial para ocorrência de mineralizações auríferas em BIFs do greenstone belt Rio das Velhas, Quadrilátero Ferrífero. Seoane et al. propõem um método para a prospecção de rochas ornamentais auxiliada por SIGs em Pernambuco. Os autores utilizaram o índice de atratividade econômico-geológica e obtiveram boa correlação entre a generalização 
dos depósitos conhecidos através desse índice e as áreas selecionadas pela integração e modelagem de dados geofísicos e de sensoriamento remoto em SIG.

Ohara et al. apresentam procedimentos para a análise integrada em SIG de dados do meio físico da região do alto-médio Rio Paraíba do Sul (SP), tendo obtido o zoneamento geoambiental e a carta de aptidão física para a implantação de obras viárias na região.

$\mathrm{O}$ artigo de Castro et al. trata do desenvolvimento de banco de dados geográficos para elaboração de mapas da morfodinâmica costeira e sensibilidade ambiental ao derramamento de óleo na costa do estado do Rio Grande do Norte.

No que concerne à Aerogeofísica, o processamento e análise de dados, integrados ou não a outros dados geológicos e de sensoriamento remoto, são assuntos centrais de oito contribuições deste fascículo. Dantas $\boldsymbol{e t} \boldsymbol{a l}$. abordam a questão da extração de informações geológicas a partir do processamento de dados aerogeofísicos do Projeto Seridó, de 1973, do qual haviam se perdido os dados digitais originais. Os autores recuperaram os dados radiométricos dos canais de $\mathrm{K}$, $\mathrm{U}$ e Th por meio da digitalização dos perfis analógicos e, apesar desta limitação, conseguiram cartografar unidades litológicas e feições estruturais com detalhamento compatível com as escalas de 1:250.000 e 1:100.000. O artigo de Blum et al. também recupera e retrabalha dados aeroradiométricos da década de 1970, neste caso do Projeto Aerogeofísico BrasilCanadá (PGBC), e delimitam os complexos granitognáissicos arqueanos de Goiás. O uso integrado de dados de sensoriamento remoto, aerogeofísicos e geológicos na definição de novas ocorrências de Urânio na região de Lagoa Real é o tema do artigo de Pascholati et al. . Trata-se de dados do Projeto São Timóteo, de 1979, que foram re-processados e analisados em conjunto com imagens TM/Landsat-5, estabelecendo um novo quadro geológico-estrutural para a região, detalhando os principais controles dos depósitos e apontando para novas ocorrências, associadas a zonas de cisalhamento de direção NW-SE. O artigo de Castro et al. mostra como a abordagem integrada de imagens de sensoriamento remoto e dados do Projeto Serra do Mar Sul, de 1978 , pode contribuir para o detalhamento das feições litoestruturais da porção central dos terrenos pré-Ordovicianos de Santa Catarina. Foram reconhecidas feições estruturais relacionadas às orogêneses Brasiliana e Rio Doce e sua importância no condicionamento de eventos tectono-magmáticos do Paleozóico. A caracterização das assinaturas gamaespectrométricas dos granitóides do Grupo Brusque e do seu uso potencial na prospecção regional de depósitos auríferos é outro resultado significativo. Este foi também o tema do artigo de Fornazzari Neto \& Fonseca, que utilizam dados re-processados do mesmo projeto aerogeofísico para determinar assinaturas gamaespectrométricas de áreas de alteração hidrotermal relacionadas às mineralizações auríferas em filões de quartzo em granitóides do Grupo Brusque em Santa Catarina. Cainzos et al. abordam a análise integrada de dados gravimétricos e aeromagnéticos na caracterização das unidades geológicas da porção centro-oriental de Cuba, por meio de técnicas de processamento e modelagem bi-dimensional de dados gravimétricos, magnéticos e geológicos; os resultados incluem o estabelecimento dos limites entre as unidades oceânicas ofiolítica e de arco vulcânico, e a unidade continental da Plataforma das Bahamas, bem como o detalhamento composicional do Arco Vulcânico Cretácico de Cuba. Madrucci et al. estabelecem as bases do modelo hidrogeológico de um aquífero fraturado em rochas pré-cambrianas no leste do estado de São Paulo, empregando informações obtidas a partir de dados de sensoriamento remoto orbital e aerogeofísicos. Os autores interpretam e analisam os elementos estruturais da área estudada, relacionando-os à produtividade de poços existentes por meio da análise espacial em ambiente SIG. No artigo de Silva et al., dados aerogeofísicos de alta resolução adquiridos sobre o greenstone belt Rio das Velhas, Quadrilátero Ferrífero, foram realçados com técnicas de processamento digital e forneceram uma melhor compreensão do controle litológico e estrutural dos depósitos auríferos da região.

Dois artigos tratam da Geoestatística aplicada à geologia e à exploração de petróleo. Remacre \& Zaparolli abordam o uso da modelagem estocástica por simulação plurigaussiana para reproduzir atributos geométricos complexos de um reservatório. Esta técnica, cujo desenvolvimento representa uma das fronteiras atuais do conhecimento científico, permite que, a partir de variáveis sobre a distribuição de litofácies em um reservatório e de funções gaussianas aleatórias, se possa estabelecer o modelo geológico mais adequado à caracterização do reservatório. Silva et al. ilustram o uso da transformada wavelet na integração espacial de dados geofísicos obtidos a partir de poços (de pouca representatividade em área e alta densidade vertical) e de dados de levantamentos sísmicos (de grande representatividade em área ou volume e baixa densidade vertical). Os autores empregam a técnica na filtragem dos dados de perfis de poços, compatibilizandoos com a escala de aquisição dos dados sísmicos, possibilitando a modelagem de fluxos em reservatórios.

O confronto entre o estado atual de desenvolvimento das Geotecnologias de forma global e o panorama de sua utilização no Brasil, revelado nos artigos que compõem este fascículo, demonstra claramente que estas pesquisas situam-se na fronteira do conhecimento científico e tecnológico. É digno de nota que os autores dos 24 artigos provém de 15 universidades públicas brasileiras, 3 instituições federais de pesquisa, 1 empresa estatal e 1 empresa privada, demonstrando a expressiva disseminação das Geotecnologias em nosso país. Vários artigos contam com a coautoria de pesquisadores estrangeiros, indicando a existência de intercâmbios internacionais e trabalhos em colaboração, com evidentes benefícios ao progresso do conhecimento científico brasileiro.

Agradecimentos Os editores vêm expressar, em nome da Sociedade Brasileira de Geologia e da Revista Brasileira de Geociências, os agradecimentos às instituições e pesquisadores que contribuíram para a publicação deste número temático. Também agradecem às empresas e instituições que contribuíram com seu apoio financeiro, de fundamental importância na publicação das ilustrações a cores, essenciais na apresentação de resultados das aplicações de geotecnologias. Colaboraram na obtenção destes apoios os Drs. Iran F. Machado, Adalene Moreira Silva, Waldir R. Paradella e Washington F. Rocha, aos quais agradecemos pelo empenho. A qualidade técnico-científica deste fascículo se deve ao criterioso trabalho, e indispensável colaboração, de numeroso grupo de consultores, especialistas nas diversas áreas, e que atuaram com rigor na revisão dos artigos submetidos. Assim, ao Corpo de Revisores, listado na abertura deste fascículo, os nossos profundos agradecimentos. Aos pesquisadores em Geotecnologias que submeteram artigos para publicação neste número temático, o nosso reconhecimento pelo excelente trabalho de pesquisa que vêm desenvolvendo e pelo esforço na promoção e difusão do conhecimento nessa área. Finalmente, os editores-convidados manifestam o seu profundo agradecimento ao Prof. Dr. Hardy Jost, pelo estímulo à organização deste temático, bem como pelo incansável apoio e desmedido esforço que possibilitou a sua publicação. 